\title{
Fat and the fate of pancreatic tumours
}

In obese people with pancreatic cancer, the many interactions between fat cells and the inflammatory microenvironment surrounding the tumour lead to below-average prognosis and chemotherapy outcome.

\section{MELEK CANAN ARKAN}

$\mathrm{T}$ he increasing prevalence of obesity will have an even greater effect on the health-care system than previously predicted, because obesity turns out to be a major risk factor for the development of cancer $^{1}$. Obese individuals have a substantially elevated risk for a type of pancreatic cancer known as pancreatic ductal adenocarcinoma, which is the fourth most-common cause of cancer-associated death ${ }^{1}$. An inflammatory microenvironment is a hallmark of cancer, but little is known about how alterations in the surrounding connective tissue (stroma) contribute to tumour initiation and progression in obesity. Writing in Cancer Discovery, Incio et al. ${ }^{2}$ report their investigation into how fat cells in the microenvironment surrounding cancer cells contribute to tumour initiation and progression in both mice and humans.

Tumour formation in the pancreas involves striking structural distortion of tissue, which is attributed to the disruption of digestiveenzyme-containing acinar cells, tissue infiltration by immune cells, a strong fibrotic response (also known as fibrosis, the formation of excess connective tissue or collagen protein around the tumour), and a higher than usual level of deposition of extracellular-matrix material. Cancer lesions in obese individuals are commonly associated with increased fat-cell (adipocyte) content compared with tumours from non-obese patients; however, the function of these fat cells in pancreatic cancer remained unclear until now.

Incio and colleagues show that, in mice, adipocytes, along with immune cells and pancreatic stellate cells, signal through the IL- $1 \beta$ protein and the AT 1 angiotensin receptor to drive migration of immune cells called neutrophils to the tumour microenvironment. This increases the inflammatory and fibrotic response in the pancreatic-cancer microenvironment in a way that results in poor response to chemotherapy and poor prognosis.

In obese mice, the tumour microenvironment was shown to contain adipocytes that are increased in both size and number, partly as a result of tumours invading the neighbouring white adipose tissues. The researchers observed an abundant fibrotic response in tumour areas that were enriched in adipocytes or located adjacent to adipose tissue. These results suggest that fibrosis is a hallmark of adipose tissue in obese subjects with pancreatic cancer, and that the accumulation of the extracellular-matrix protein collagen, a component of the fibrotic response, in the vicinity of fat cells is a prominent characteristic of obesity. Incio and colleagues also found that adipocyte infiltration into the tumour microenvironment correlates with worse prognosis and treatment outcome in patients.

The authors hypothesized that, in people with pancreatic cancer, obesity-associated adipocyte accumulation increases fibrosis, promotes tumour progression and hinders the delivery and efficacy of chemotherapeutics. When they checked the percentage of perfused blood vessels in a given area of mouse tumour, they found that it was significantly reduced in obese animals. To determine whether impeded perfusion through blood vessels is responsible for inefficient delivery of chemotherapeutic agents, the authors measured the uptake of the chemotherapy drug 5-fluorouracil in mice. Obesity significantly decreased tumour uptake of the drug compared with uptake in non-obese control animals, thereby reducing the chemotherapy's efficacy (Fig. 1).

Chronic fibrosis is thought to have a crucial role in enhancing tumour growth and in attenuating drug delivery. However, in previous studies, inhibition of chronic fibrosis by either inhibitor compounds $s^{3}$ or genetic mutations $s^{3,4}$ resulted in increased immunosuppression, accelerated tumour growth and decreased survival, implying that tumour stroma may be restrictive to tumour growth.

By contrast, Incio et al. show that inhibition of the major pro-fibrotic pathway of AT1 signalling in mice inhibited tumour progression. The authors propose that migration into the tissue of tumour-associated neutrophils and IL- $1 \beta$ production are leading drivers in the regulation of tumour growth in this context, although changes in vascular perfusion due to reduced blood pressure also play a minor part. When the authors depleted neutrophils or blocked the activity of IL- $1 \beta$ using antibody treatment, the immunosuppressive microenvironment was reshaped and the progression of pancreatic cancer was reduced.

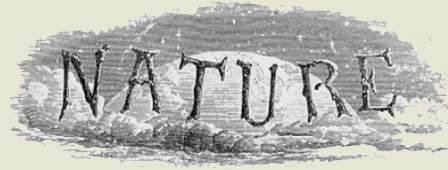

50 Years Ago

'We wuz robbed' - The World Cup which has recently been enacted in Britain may have been fun to watch, but there is no question that it was a thoroughly badly designed experiment ... The mere fact that a Poisson distribution can describe so well the distribution of scores by individual teams goes a long way to suggest that the teams were much of a muchness in talent and their scores were independent of each other. From this point of view, the decision that the outcome of the whole competition should depend on the outcome of a single game between the two so-called finalists was as much of a farce as a great many West German supporters already know it to have been ... If, for example, it were agreed that ... no team should be declared the winner until its score exceeds that of its opponent by three standard deviations of Poisson distribution, it might be necessary to design the game of football so that it would be practicable for one side to score 100 goals or so ... Such a change could easily be brought about, possibly by widening the goalposts or by abolishing goalkeepers.

From Nature 13 August 1966

\section{Years Ago}

The History of the Family. By Prof. $\mathrm{W}$. Goodsell - In what sense is it right to speak of the history of the family? ... Can it be said to have a history? ... Some such questions as these arise in one's mind as one takes up Prof. Goodsell's book ... even a casual reader will be struck by a want of precise references in certain of the chapters ... Where is the "weight of evidence" which shows that polygamy is unpopular among savage women? The author gives several reasons why we condemn it, but there is surely room for doubt...

From Nature 10 August 1916 
Tumour in non-obese context

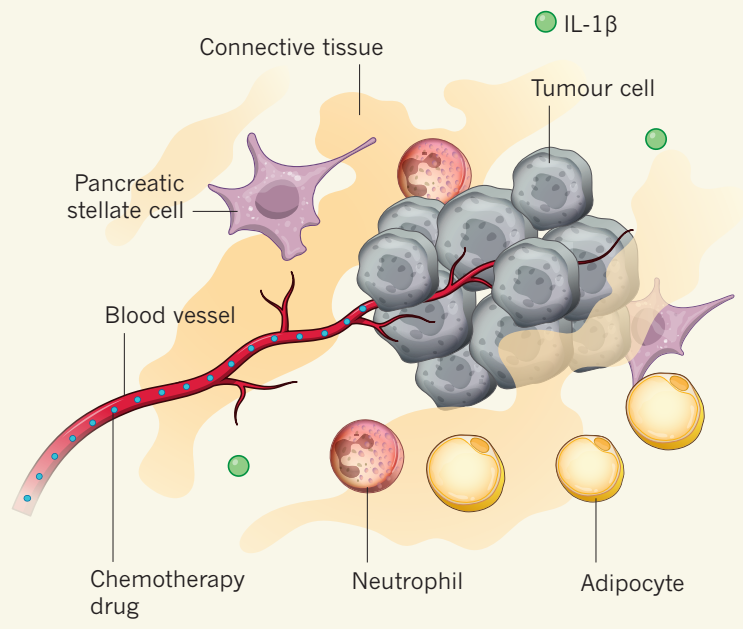

Figure 1 | Fat cells remodel the microenvironment around

tumours. Tumours are perfused with blood vessels, which allow chemotherapy drugs to enter. Incio et al. ${ }^{2}$ report that, in the context of obesity, access to pancreatic tumours is restricted by poor tumour bloodvessel perfusion, leading to a decreased response by tumour cells to chemotherapeutic drugs. In obesity, there is an increase in pancreatic stellate cells, immune cells such as neutrophils and IL- $1 \beta$ signalling molecules, as
Tumour in obese context

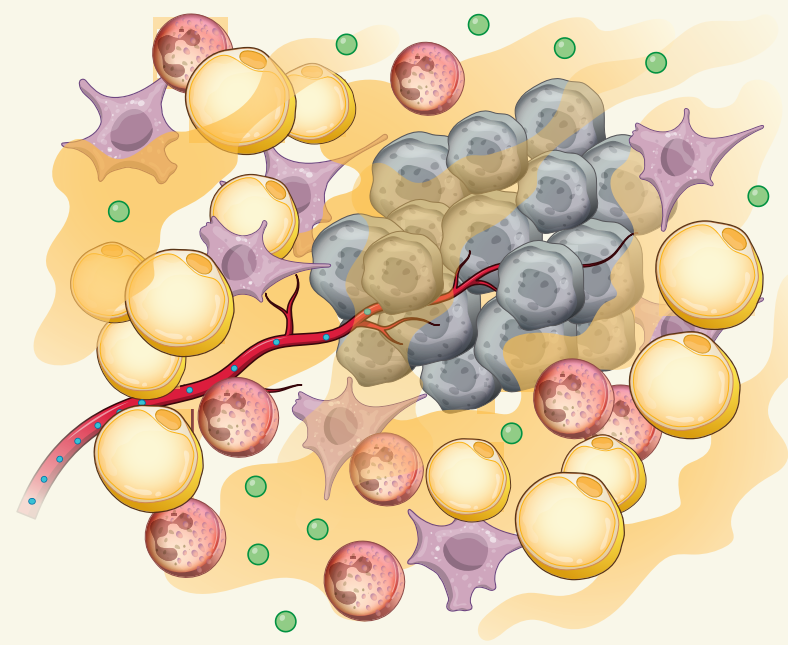

well as larger fat cells (adipocytes). The denser cellular microenvironment seen in obesity puts extra mechanical tension on the tissue and may restrict blood-vessel perfusion. This mechanical tension arises because of the signalling crosstalk between adipocytes, neutrophils, pancreatic stellate cells and other components of the tissue microenvironment. This crosstalk leads to an increase in inflammatory cells such as neutrophils and excess fibrous connective tissue in the vicinity of the tumour.
When experimentally targeting AT1 signalling in mice, other processes downstream of AT1 signalling - such as the epithelial-tomesenchymal cell transition or adipocyte differentiation - might also be affected, and it is possible that these processes are responsible for the decrease in obesity-associated tumour progression.

Even though the authors correlated fibrotic response with tumour size, it is difficult to judge whether the fibrotic response or tumour growth comes first, because decreased tumour progression will eventually result in decreased immune-cell infiltration into the tumour microenvironment and decreased fibrosis. Either way, the results of this study and oth$\mathrm{ers}^{3,4}$ reinforce the need for further evaluation of the functional contribution of fibrosis in the initiation and progression of pancreatic cancer, especially in obesity.

Cellular alterations induced by mechanical forces are becoming more widely recognized as having a role in various diseases ${ }^{5}$. Homeostasis in the balance between internal and external forces on cells (the state of physical tension known as tensional homeostasis) can regulate apoptotic cell death, cell proliferation, adhesion and migration, and its deregulation could result in increased susceptibility to cancer. In addition, physical cues from the pressure exerted by solid-tissue components of the tumour microenvironment can compress blood vessels, causing poor tumour perfusion ${ }^{6}$.

Incio and colleagues showed that treatment with the AT1 blocker losartan can reduce mechanical stress on cells and decrease tumour growth in mice with pancreatic cancer. More research is needed to investigate the type of transcriptional switch that tensional homeostasis induces in the dense cellular milieu of the tumour microenvironment in obesity. Inhibition of the mechanical forces acting on cells in pancreatic cancer may provide further clues for future clinical treatment. Normalizing the tumour extracellular matrix by reducing matrix stiffness may be more effective and safer than trying to delete stromal components directly.

The relationship between fat cells and stemcell regulation is another key question. Mature white adipocytes - fat stores that control energy metabolism - respond to nutritional and hormonal cues through the secretion of signalling proteins. Studies point to white adipose fat having functions in tissue regeneration and stem-cell regulation, placing fat cells at the centre of multiple aspects of cancer progression ${ }^{7}$. Mesenchymal stem cells or stromal stem cells contribute substantially to adipocyte generation. Mechanical stress is also a trigger for the expansion of some stem-cell populations $^{8}$.

It would be interesting to determine the origin of adipocytes in the pancreas, their fate and phenotype (whether the cells form white or brown fat, as well as the type of component they secrete). Other potential topics for investigation include studying the contribution of adipocyte-derived signals that recruit and possibly polarize immune-cell differentiation; the function of adipocyte invasion during tumour formation; and the role of malfunctioning energy metabolism in obesity.

Incio and colleagues' work provides a plausible cellular and molecular explanation for increased adipocyte interaction with the cells of the pro-inflammatory and pro-fibrotic tumour microenvironment that accelerates disease progression and hampers therapy. Is systemically targeting tumour-associated neutrophils, pancreatic stellate cells or adipocytes feasible without causing collateral damage to host functions? Such damage could be a major challenge to successful direct translation of the current findings to the clinic. Only time will tell whether targeting IL- $1 \beta$ or neutrophils could offer opportunities for successful therapeutic intervention. Of course, the best preventive approach in the meantime is to eat a healthy diet and to exercise.

Melek Canan Arkan is at the Institute of Biochemistry II, Goethe University, Frankfurt 60590, and the Institute for Tumor Biology and Experimental Therapy, Georg-Speyer

Haus, Frankfurt 60596, Germany. e-mail:arkan@med.uni-frankfurt.de

1. Giovannucci, E. \& Michaud, D. Gastroenterology 132, 2208-2225 (2007).

2. Incio, J. et al. Cancer Discov. http://dx.doi. org/10.1158/2159-8290.CD-15-1177 (2016).

3. Rhim, A. D. et al. Cancer Cell 25, 735-747 (2014).

4. Özdemir, B. C. et al. Cancer Cell 25, 719-734 (2014).

5. DuFort, C. C., Paszek, M. J. \& Weaver, V. M. Nature Rev. Mol. Cell Biol. 12, 308-319 (2011).

6. Provenzano, P. P. \& Hingorani, S. R. Br. J. Cancer 108, 1-8 (2013).

7. Shook, B. et al. Annu. Rev. Cell Dev. Biol. http://dx.doi.org/10.1146/annurevcellbio-111315-125426 (2016).

8. Guilak, F. et al. Cell Stem Cell 5, 17-26 (2009).

This article was published online on 3 August 2016. 